\title{
Carica papaya's Varieties Improved Obesity, Insulin Resistance and Atherogenic Risk in Rats Fed with Oxidised Palm Oil and Sucrose Diet
}

\author{
Etaga Noel Babayana ${ }^{1}$, Selakong Nzekuie Quelie ${ }^{1}$, Etoundi Omgba Cunegonde Blanche ${ }^{2}$, \\ Mekoulou Ndongo Jerson ${ }^{1}$, Bika Lélé Claude Élisé ${ }^{1}$, Aicha De Nkainsa ${ }^{1}$, Ngaha Marie Ide ${ }^{1}$, \\ Bilog Nadine Carole ${ }^{1}$, Bogning Zangueu Calvin ${ }^{1}$, Mbock Armel Junior ${ }^{1}$, \\ Endougou Effa Anne Marie ${ }^{1}$, Dongmo Alain Bernard ${ }^{1}$, Bongue Bienvenu ${ }^{3}$, \\ Mandengue Samuel Honoré ${ }^{1}$, Ayina Ayina Clarisse Noel ${ }^{1, *}$ \\ ${ }^{1}$ Department of Animal and Biology, University of Douala, Douala, Cameroun \\ ${ }^{2}$ Department of Biochemistry, University of Douala, Douala, Cameroun \\ ${ }^{3}$ Laboratoire SAINBIOSE INSERM U1059, Université Jean Monnet, Saint Etienne, France
}

\begin{abstract}
Email address:
etaganoel@yahoo.fr (E. N. Babayana), qselakong@yahoo.fr (S. N. Quelie), blancheetoundi@hotmail.fr (E. O. C. Blanche), meckjerson@yahoo.fr (M. N. Jerson), claudebika@gmail.com (B. L. C. Élisé), aichankansa615@yahoo.com (A. De Nkainsa), ngahamarie@yahoo.fr (N. M. Ide), billycarolle10@yahoo.fr (B. N. Carole), calvinbongz@yahoo.fr (B. Z. Calvin), armeljunior_mbock@yahoo.fr (M. A. Junior), eff_anmary@yahoo.fr (E. E. A. Marie), alainberd@yahoo.fr (D. A. Bernard), bienvenu.bongue@univ-st-etienne.fr (B. Bienvenu), shmandengue@yahoo.fr (M. S. Honoré), c_ayina@yahoo.fr (A. A. C. Noel) ${ }^{*}$ Corresponding author
\end{abstract}

\section{To cite this article:}

Etaga Noel Babayana, Selakong Nzekuie Quelie, Etoundi Omgba Cunegonde Blanche, Mekoulou Ndongo Jerson, Bika Lélé Claude Élisé, Aicha De Nkainsa, Ngaha Marie Ide, Bilog Nadine Carole, Bogning Zangueu Calvin, Mbock Armel Junior, Endougou Effa Anne Marie, Dongmo Alain Bernard, Bongue Bienvenu, Mandengue Samuel Honoré, Ayina Ayina Clarisse Noel. Carica papaya's Varieties Improved Obesity, Insulin Resistance and Atherogenic Risk in Rats Fed with Oxidised Palm Oil and Sucrose Diet. International Journal of Nutrition and Food Sciences. Vol. 10, No. 5, 2021, pp. 108-116. doi: 10.11648/j.ijnfs.20211005.13

Received: August 11, 2021; Accepted: September 2, 2021; Published: October 12, 2021

\begin{abstract}
In the recent years, obesity has become more prevalent owing to increase habit sedentary. According to many studies, physical activities and nutritherapy could be complementary solutionto this problem. Pawpaw (Carica papaya) is a tropical fruit highly consumed for its flavour and nutritive properties. The present studywas carried out to investigate the effect of some pawpaw's varieties' juice on obesity, insulin resistance and atherogenic riskin albino rats under oxidised palm oil and sucrose diet. Healthy males and females Wistar rats received during 13 weeks every day a supplement of oxidised palm oil $(25 \%)$ sucrose $(25 \%)$. At the end of this period, animals presenting obesity, insulin resistance and glucose intolerancecharacteristics were randomly separated into 6 groups. Three of these groups (satellite control groups (G III, G IV and $\mathrm{G} \mathrm{V}$ ) returned to standard diet and received a supplement of smooth cayenne and spanich variety of pawpaw (1 ml/100g), while thethree others groups (OPOS control groups G II, G VI and G VII) continued with oxidised palm oil and sucrose diet (OPOS), and received a supplement of smooth cayenne and spanich variety of pawpaw ( $1 \mathrm{ml} / 100 \mathrm{~g})$, respectively during 12 weeks. Oral Glucose Tolerance Test (OGTT) was performed and insulin sensitivity was assessed by performing insulin tolerance test, then determining insulin index (Kitt). Body weight, Lee index, naso-anal length, abdominal fat mass, BMI, faeces relative weight and lipid profilewere measured; Atherogenic risk was calculated. OPOS diet induced overall obesity, abdominal obesity, insulin resistance, dyslipidemia and atherogenic risk, and thenreduced Faeces relative weight in animals $(\mathrm{P}<0.001$ respectively). Treatment with two varieties of pawpaw fresh juice significantly $(\mathrm{P}<0.001)$ reduced Lee index, nasoanal length, abdominal fat mass andBMI, and increased faeces weight; Glucose tolerance and insulin sensitivity were restored; the lipid profile was improved and the atherogenic risk eliminated both in males and females. These results highlight the obesogenic, hyperglycaemic and dyslipidemic characters of OPOS diet, and show the benefits of pawpaw fresh juice on these metabolic diseases. With complementary studies, we will recommend the consumption of this fruit as complementary solution
\end{abstract}


for theprevention and/or the management of the cardiometabolic risk.

Keywords: Pawpaw (Carica papaya), Oxidised Palm Oil, Sucrose, Obesity, Insulin Resistance

\section{Introduction}

Sedentary lifestyle and excess caloric intake from a diet high in fats and sugars have made obesity international public health problems [1]. Obesity acts as a stressing agent both in adipose metabolism and in metabolic organs, including the liver, muscle and pancreas resulting in insulin resistance and type II diabetes mellitus [2]. Obesity reduce life expectancy of up to 7 years compared with normal weight individuals and predict the future development of metabolic syndrome, type 2 diabetes mellitus and cardiovascular diseases [3]. Excess adiposity and poor cardio respiratory fitness drive the epidemic of type 2 diabetes mellitus and early cardiovascular [4]. Cardiovascular complications are the main cause of morbidity in obese, insulin resistance and type 2 diabetes mellitus. Consequently, obesity and its comorbidities, characterized by hyperglycaemia, insulin resistance, dyslipidemia, low-grade systemic inflammation, elevated plasma triacylglycerol and total cholesterol, may lead to the onset of metabolic syndrome, a serious threat to the population health $[5,6]$. The major cause of obesity is the chronic excessive energy intake from calorie-dense foods, such as high fat foods that contain greater caloric content than the carbohydrate or protein constituents coupled with decreased energy expenditure [6, 7]. It's recognized that calorie restriction limits the amount of daily calorie intake to $60-70 \%$ of ad libitum feeding. In human and mammals long-termed calorie restriction results in sustained benefits on major risk factors for atherosclerosis, type 2 diabetes and inflammation [8]. The incidence of obesity is increasing all over the world. For weight loss, surgery seems an option when other treatment modalities have failed [9]. Even if at present time bariatric surgery allows to have notable results on the loss of weight and the survival of obese individuals, it is necessary to improve the prevention of the metabolic complications the medical care and to adapt best strategy aiming to manage obesity and its comorbidities. Pawpaw is a tropical fruit probably favorable in obesity and dyslipidemia treatment, as it contains a high amount of papaïn, chymopapaïne, lipase etc; lipase is efficient on lipolysis and diminishing the severity of cardiovascular syndromes [10]. Fresh fruits are known for their defensive impact against cardiovascular diseases [10]. They have the benefit of containing high amounts of specific dietary fiber, which decreases cholesterol uptake by the organism [10]. Fat binding capacity (FBC), important for the detection of anti-obesity property in food, was very high in pawpaw. A couple of clinical investigations demonstrate the helpful impacts of papain in stoutness treatment [10]. This study was therefore, conducted to investigate ameliorative effects of two varieties of pawpaw fruit on obesity and insulin resistance in rats.

\section{Materials and Methods}

\subsection{Juice Preparation}

Pawpaw fruits collection and juice preparation

Two most cultivated varieties of fresh Solo number 8 pawpaw fruits Carica papaya (L). (Caricaceae): weth yellow-orange flesh oblong form (hermaphrodites feet), and round form (females feet) were collected from Djombe-Penja Subdivision (Littoral region - Cameroon), identified at the Cameroon National Herbarium, where a specimen has been deposited under the number $66220 / \mathrm{HNC}$. The fruits were washed and peeled; then, the edible portion was cut into tiny pieces and placed in a semi-industrial juice extractor (Moulinex). Juicing process somehow preserved fibers. After processing, the juice obtained was lyophilized.

\subsection{Animals}

Eighty four adult male and female Wistar rats (forty two males and forty two females, 8 weeks old) were obtained from experimental animal centre, Faculty of Science of University of Douala. The rats were retained under controlled states of temperature $\left(23 \pm 2^{\circ} \mathrm{C}\right)$, humidity $(50 \pm 5 \%)$ and $12 \mathrm{~h}$ light-dim cycle. Rats were acclimatized for a week before beginning the experiment. They were kept in sanitized polypropylene cages containing sterile husk as bedding with free access to standard pellets as basal diet and water ad libitum. All procedures approved by with the European Union on Animal Care (CEE Council 86/609) guidelines adopted by the Institutional Ethics Committee of the Cameroon Ministry of Scientific Research and Technology Innovation.

\subsection{Experimental Design}

After one-week of acclimatization, rats (both sexes) were divided randomly into two main groups. G I (control, $n=6$, each sex) that was kept on normal diet. OPOS group $(n=36$, each sex) that was fed on OPOS (oxidised palm oil and sucrose) (1 kg ration contained $250 \mathrm{~g}$ oxidized oil, $250 \mathrm{~g}$ of sucrose and $500 \mathrm{~g}$ of standard diet (corn $40 \%$, soybeans $20 \%$, fish $20 \%$, peanut $8 \%$, wheat $7 \%$, cotton $2 \%$, bone $2 \%$ and vitamins $1 \%$ ) according to the modified method of Ngueguim et al. [11]. Animals in this group gained free access to water for 13 weeks until the point that obesity was confirmed by low faeces weight, high BMI, high Lee index, high abdominal circumference and low naso-anal lenght. After the 13 week of obesity induction, OPOS group were divided into 6 obese groups (each group contained 6 rats) which were treated for 13 more weeks as follow: Untreated obese group (G II) that continued with OPOSdiet and receiveddistilled water $(1 \mathrm{~mL} / 100 \mathrm{~g})$, the $2^{\text {nd }}$ obese group (G III) received normal diet and distilled water $(1 \mathrm{~mL} / 100 \mathrm{~g})$, the $3^{\text {rd }}$ obese group (G IV) 
received normal diet and $1 \mathrm{~mL} / 100 \mathrm{~g}$ of pawpaw fruit juice of oblong form, $4^{\text {th }}$ obese group $(\mathrm{G} \mathrm{V})$ continued with OPOS diet and received $1 \mathrm{~mL} / 100 \mathrm{~g}$ of pawpaw fruit juice of oblong form, the $5^{\text {th }}$ obese group (G VI) received normal diet and 1 $\mathrm{mL} / 100 \mathrm{~g}$ of pawpaw fruit juice of round form, $6^{\text {th }}$ obese group continued with OPOS diet and received $1 \mathrm{~mL} / 100 \mathrm{~g}$ of pawpaw fruit juice of round form (G VII).

\subsection{Measurements}

Anthropometrical parameters of each group were recorded weekly throughout the experimental period (26 weeks). Serum glucose level of each rat were assessed at the beginning of the experiment $\left(\mathrm{W}_{0}\right)$ and then after thirteen weeks $\left(\mathrm{W}_{13}\right)$ and twenty six weeks $\left(\mathrm{W}_{26}\right)$; and to evaluate insulin resistance in this period, insulin tolerance test was performed [12] and insulin sensitivity index calculated [12]. After $12 \mathrm{~h}$, of fasting, the glycaemia was evaluate at $0 \mathrm{~h}$ and animals received $2 \mathrm{UI} / \mathrm{kg}$ of insulin and blood was obtained from tail at 12, 20, 30 and $60 \mathrm{~min}$ after insulin injection; serum glucose was measured and $\mathrm{K}_{\text {itt }}$ value was obtained using the following formula:

$\mathrm{K}_{\mathrm{ITT}}=(\mathrm{C} 0-\mathrm{Cmin}) / \mathrm{C} 0=$ concentration at $0 \mathrm{~h}$, minimal concentration.

Twenty-four hours after the last treatment, oral glucose tolerance test was evaluate according to the method of Tritos and Manzoros [12] and the animals were scarified through terminal exsanguination under ketamine $(80 \mathrm{mg} / \mathrm{kg})$ and xylazine $(10 \mathrm{mg} / \mathrm{kg})$ anaesthesia. Fasting blood samples were collected through cardiac puncture into labelled tubes and the sera were stored at $-80^{\circ} \mathrm{C}$ for blood lipid analyses. Then, the rats were sacrificed and dissected, certain organs and abdominal fats harvested and weighted.

\subsection{Serum Biochemical Assays}

Serum total cholesterol (TC), triacylglycerides (TG) andHDLcholesterol weremeasured using a spectrometer, then atherogenic index was determined by the ratio LDLcholesterol/HDL-c [13].

\subsection{Statistical Analysis}

All data were expressed as mean \pm standard error mean. Statistical significance was determined by applying one-way ANOVA with post-test turkey using statistics software package (graph pad prism V. 8. 01. Difference significant at $P$ value $<0.05$.

\section{Results}

\subsection{Effects of Supplement of Oxidised Palm Oil and Sucrose on Anthropometrical Parameter}

After 13 weeks of induction, animals submitted to food supplemented with oxidised palm oil $(25 \%)$ and $25 \%$ of sucrose (OPOS) in both sex presented an increase of abdominal fat mass more than $78.83 \%(8.54 \pm 0.68$ vs $2.68 \pm 0.26,10.96 \pm 0.77$ vs $2.32 \pm 0.32$; $\mathrm{P}<0.001)$, BMI $26.98 \%(0.63 \pm 0.02$ vs $0.46 \pm 0.02$,
$0.6 \pm 0.02$ vs $0.46 \pm 0.02 ; \mathrm{P}<0.001)$, abdominal circumference $47.57 \%$ (35.92 \pm 0.72 vs $24.34 \pm 0.28,35.92 \pm 0.87$ vs $24.32 \pm 0.72$; $\mathrm{P}<0$. 001), Lee index $15.63 \% \quad(0.32 \pm 0.01$ vs $0.27 \pm 0.00$, $0.32 \pm 0.01$ vs $0.27 \pm 0.01 ; \mathrm{P}<0.001)$, liver and kidney relative weight $61.31 \%(6.1 \pm 0.4$ vs $2.36 \pm 0.1,5.25 \pm 0.47$ vs $2.98 \pm 0.16$; $\mathrm{P}<0.001)$ respectively for males and females. OPOS also induced a decrease of naso-anal length $20.18 \%$ (19.06 \pm 0.36 vs $23.88 \pm 0.36,18.1 \pm 0.06$ vs $20.4 \pm 0.24 ; \mathrm{P}<0.001$ respectively for males and females), faeces weight $(\mathrm{P}<0.001)$ in control group compared to the OPOS groups (G II).

\subsection{Effects of Supplement of Oxidised Palm Oil and Sucrose on Insulin Sensibility}

After 13 weeks of induction, a subcutaneous injection of insulin $(2 \mathrm{UI} / \mathrm{kg})$ to animal receiving OPOS diet revealed that glycaemia remained significantly higher throughout the experimental period (Table 1) in comparison with rats receiving standard diet (in both sex). The insulin sensitivity index was 0.44 and 0.51 (respectively for males and females) for normal rats versus 0.16 and 0.09 (respectively for males and females) for animals receiving OPOS diet. Insulin sensitivity index was significantly decreased of 63.64 and $82.35 \%$ (respectively for males and females) for animals receiving OPOS diet as compared to the normal control.

\subsection{Effects of Supplement of Oxidised Palm Oil and Sucrose on Blood Lipids and Atherogenic Risk}

13 weeks of food supplementation with oxidised palm oil (25\%) and $25 \%$ of sucrose (OPOS) in the both sex induced an increase of triglyceride (TG) for more than $47.46 \%$ $(287 \pm 16.75$ vs $150.8 \pm 10.98,277.1 \pm 12.06$ vs $151.1 \pm 9.40$; $\mathrm{P}<0.001)$, total cholesterol (TC) $51.50 \% \quad(810.3 \pm 83.29$ vs $393 \pm 2.29, \quad 577.6 \pm 26.03$ vs $387.6 \pm 5.31 ; \mathrm{P}<0.001)$, LDL-c $61.55 \%$ ( $704.5 \pm 101.5$ vs $270.9 \pm 2.7,457 \pm 15.9$ vs $269.8 \pm 8.38$; $\mathrm{P}<0.001$ ), atherogenic index (AI) $78.17 \%(19.47 \pm 2.74$ vs $4.25 \pm 0.06,12.79 \pm 0.21$ vs $4.54 \pm 0.22 ; \mathrm{P}<0.001)$ respectively for males and females. OPOS diet also induced a decrease of HDL-c of $55.12 \%$ compared to normal control.

\subsection{Effects of C. Papaya on Anthropometrical Parameter}

For 13 weeks, obese animals were treated with pawpaw juice, which induced significant decreases of abdominal fat mass (50.35 and 50.75\%), BMI (21.31 \%and $21.54 \%$ ), abdominal circumference $(27.24 \%$ and $23.48 \%$ ) and Lee index (17.65\% and $14.71 \%)$ respectively for oblong and round form (G IV and G VI) compared to G III. Lee index and Naso-anal length remained significantly different between negative control group (G I) and the groups treated with pawpaw juice extract $(\mathrm{P}<0.001$ respectively). However, pawpaw juice significantly increased faeces' weight in treated animals $(\mathrm{P}<0.001)$, comparatively to obese untreated. No significant difference of pawpaw juice effect $(\mathrm{P}>0.05)$ was observed neither between oblong and round form groups, neither between animals fed with normal diet and those fed with OPOS, nor between males and females (Table 1). 
Table 1. Effects of concomitant administration of Carica papaya (Oblong and Round form) juice extract and oxidised palm oil + sucrose on anthropometrical parameters in rats.

\begin{tabular}{|c|c|c|c|c|c|c|c|c|}
\hline \multirow{2}{*}{ Parameters } & \multirow{2}{*}{ Weeks } & \multicolumn{7}{|l|}{ MALES } \\
\hline & & G I & G II & G III & G IV & G V & G VI & G VII \\
\hline \multirow{3}{*}{$\mathrm{AC}(\mathrm{cm})$} & W 0 & $13.86 \pm 0.10$ & $14.26 \pm 0.08$ & $14.28 \pm 0.12$ & $14.54 \pm 0.06$ & $14.72 \pm 0.04$ & $14.69 \pm 0.06$ & $14.67 \pm 0.3$ \\
\hline & W13 & $24.04 \pm 0.25$ & $35.76 \pm 0.97^{\mathrm{a} 3}$ & $35.68 \pm 0.92^{\mathrm{a} 3}$ & $35.34 \pm 0.34^{\mathrm{a} 3}$ & $36.12 \pm 0.51^{\mathrm{a} 3}$ & $36.31 \pm 0.87^{\mathrm{a} 3}$ & $36.07 \pm 0.73^{\mathrm{a} 3}$ \\
\hline & W26 & $24.34 \pm 0.28$ & $36.68 \pm 0.72^{\mathrm{a} 3}$ & $35.12 \pm 0.93^{\mathrm{a} 3}$ & $27.76 \pm 1.54^{\mathrm{blc} 3}$ & $31.12 \pm 1.06^{\mathrm{cl}}$ & $28.77 \pm 1.22^{\mathrm{b} 1 \mathrm{c} 3}$ & $32.22 \pm 1.46$ \\
\hline \multirow{3}{*}{ NAL (cm) } & W 0 & $17.38 \pm 0.27$ & $17.06 \pm 0.12$ & $16.90 \pm 0.3$ & $17.12 \pm 0.22$ & $17.82 \pm 0.25$ & $17.61 \pm 0.23$ & $18.29 \pm 0.55$ \\
\hline & W13 & $22.74 \pm 0.56$ & $19.10 \pm 0.38^{\mathrm{a} 3}$ & $18.92 \pm 0.26^{\mathrm{a} 3}$ & $20.10 \pm 0.47^{\mathrm{a} 3}$ & $19.10 \pm 0.25^{\mathrm{a} 3}$ & $18.88 \pm 0.47^{\mathrm{a} 3}$ & $18.97 \pm 0.24^{\mathrm{a} 3}$ \\
\hline & W26 & $23.88 \pm 0.36$ & $19.06 \pm 0.36^{\mathrm{a} 3}$ & $19.50 \pm 0.15^{\mathrm{a} 3}$ & $20.70 \pm 0.80$ & $19.40 \pm 0.21^{\mathrm{a} 3}$ & $20.20 \pm 0.37^{\mathrm{a} 1}$ & $19.75 \pm 0.40^{\mathrm{a} 3}$ \\
\hline \multirow{3}{*}{ AFM (g) } & W 0 & $1.28 \pm 0.57$ & $0.34 \pm 0.07$ & $1.24 \pm 0.28$ & $0.36 \pm 0.03$ & $1.16 \pm 0.14$ & $1.16 \pm 0.18$ & $1.192 \pm 0.07$ \\
\hline & W13 & $1.63 \pm 0.27$ & $7.19 \pm 0.38^{\mathrm{a} 3}$ & $8.97 \pm 1.05^{\mathrm{a} 3}$ & $7.35 \pm 0.26^{\mathrm{a} 3}$ & $8.3 \pm 0.40^{\mathrm{a} 3}$ & $7.78 \pm 0.80^{\mathrm{a} 3}$ & $8.10 \pm 0.80^{\mathrm{a} 3}$ \\
\hline & W26 & $2.68 \pm 0.26$ & $10.05 \pm 0.57^{\mathrm{a} 3}$ & $8.54 \pm 0.68^{\mathrm{a} 3}$ & $4.99 \pm 0.14^{\mathrm{a} 3 \mathrm{~b} 3 \mathrm{c} 3}$ & $5.38 \pm 0.19^{\text {азьзс } 3}$ & $4.95 \pm 0.18^{\text {a3b3c3 }}$ & $5.29 \pm 0.22^{\mathrm{a} 3 \mathrm{~b} 3 \mathrm{c} 3}$ \\
\hline \multirow{3}{*}{$\operatorname{BMI}\left(\mathrm{g} / \mathrm{cm}^{2}\right)$} & W 0 & $0.45 \pm 0.02$ & $0.48 \pm 0.01$ & $0.49 \pm 0.02$ & $0.49 \pm 0.02$ & $0.46 \pm 0.01$ & $0.47 \pm 0.1$ & $0.44 \pm 0.03$ \\
\hline & W13 & $0.47 \pm 0.02$ & $0.68 \pm 0.04^{\mathrm{a} 3}$ & $0.67 \pm 0.03^{\mathrm{a} 3}$ & $0.65 \pm 0.02^{\mathrm{a} 3}$ & $0.61 \pm 0.02^{\mathrm{a} 3}$ & $0.66 \pm 0.04^{\mathrm{a} 3}$ & $0.61 \pm 0.04^{\mathrm{a} 3}$ \\
\hline & W26 & $0.46 \pm 0.01$ & $0.69 \pm 0.03^{\mathrm{a} 3}$ & $0.63 \pm 0.01^{\mathrm{a} 3}$ & $0.49 \pm 0.02^{\mathrm{b} 2 \mathrm{c} 2}$ & $0.53 \pm 0.01^{\mathrm{b} 2 \mathrm{c} 2}$ & $0.50 \pm 0.01^{\mathrm{b} 2 \mathrm{c} 2}$ & $0.53 \pm 0.01^{\mathrm{b} 2 \mathrm{c} 2}$ \\
\hline \multirow{3}{*}{$\begin{array}{l}\text { Lee Index } \\
\left(\mathrm{g} / \mathrm{cm}^{3}\right)\end{array}$} & W 0 & $0.3 \pm 0.01$ & $0.3 \pm 0.00$ & $0.3 \pm 0.00$ & $0.3 \pm 0.00$ & $0.29 \pm 0.00$ & $0.29 \pm 0.00$ & $0.29 \pm 0.01$ \\
\hline & W13 & $0.27 \pm 0.01$ & $0.33 \pm 0.01^{\mathrm{a} 3}$ & $0.33 \pm 0.00^{\mathrm{a} 3}$ & $0.32 \pm 0.00^{\mathrm{a} 3}$ & $0.32 \pm 0.00^{\mathrm{a} 3}$ & $0.33 \pm 0.01^{\mathrm{a} 3}$ & $0.32 \pm 0.01^{\mathrm{a} 3}$ \\
\hline & W26 & $0.27 \pm 0.00$ & $0.34 \pm 0.01^{\mathrm{a} 3}$ & $0.32 \pm 0.00^{\mathrm{a} 3}$ & $0.28 \pm 0.01^{\mathrm{b} 3 \mathrm{c} 3}$ & $0.3 \pm 0.00^{\mathrm{b} 2}$ & $0.29 \pm 0.00^{\mathrm{b} 3 \mathrm{c} 3}$ & $0.3 \pm 0.00^{\mathrm{b} 2}$ \\
\hline \multirow{3}{*}{ FW (g/100g) } & W 0 & $1.26 \pm 0.11$ & $1.19 \pm 0.02$ & $1.5 \pm 0.18$ & $0.97 \pm 0.11$ & $1.19 \pm 0.04$ & $1.32 \pm 0.14$ & $1.21 \pm 0.09$ \\
\hline & W13 & $2.28 \pm 0.11$ & $1.24 \pm 0.07^{\mathrm{a} 3}$ & $1.18 \pm 0.10^{\mathrm{a} 3}$ & $1.13 \pm 0.04^{\mathrm{a} 3}$ & $1.26 \pm 0.11^{\mathrm{a} 3}$ & $1.16 \pm 0.14^{\mathrm{a} 3}$ & $1.21 \pm 0.06^{\mathrm{a} 3}$ \\
\hline & W26 & $2.42 \pm 0.09$ & $1.09 \pm 0.10^{\mathrm{a} 3}$ & $1.6 \pm 0.06^{\mathrm{a} 3}$ & $2.19 \pm 0.07^{\mathrm{b} 3 \mathrm{c} 2}$ & $2.52 \pm 0.04^{\mathrm{b} 3 \mathrm{c} 2}$ & $2.24 \pm 0.13^{\mathrm{b} 3 \mathrm{c} 2}$ & $2.35 \pm 0.07^{\mathrm{b} 3 \mathrm{c} 2}$ \\
\hline \multirow{2}{*}{ Parameters } & \multirow{2}{*}{ Weeks } & \multicolumn{7}{|l|}{ FEMALES } \\
\hline & & G I & G II & G III & G IV & G V & G VI & G VII \\
\hline \multirow{3}{*}{$\mathrm{AC}(\mathrm{cm})$} & W 0 & $14.50 \pm 0.18$ & $14.13 \pm 0.04$ & $14.37 \pm 0.11$ & $14.26 \pm 0.14$ & $14.67 \pm 0.19$ & $14.36 \pm 0.13$ & $14.28 \pm 0.35$ \\
\hline & W13 & $24.70 \pm 0.26$ & $36.33 \pm 0.89^{\mathrm{a} 3}$ & $35.74 \pm 0.70^{\mathrm{a} 3}$ & $34.66 \pm 0.52^{\mathrm{a} 3}$ & $34.87 \pm 0.17^{\mathrm{a} 3}$ & $35.16 \pm 0.44^{\mathrm{a} 3}$ & $36.84 \pm 0.86^{\mathrm{a} 3}$ \\
\hline & W26 & $24.72 \pm 0.23$ & $38.97 \pm 0.40^{\mathrm{a} 3}$ & $35.92 \pm 0.87^{\mathrm{a} 3}$ & $30.06 \pm 0.61^{\mathrm{b} 3 \mathrm{c} 3}$ & $32.04 \pm 0.98^{\mathrm{b} 3 \mathrm{c} 3}$ & $27.53 \pm 1.09^{\mathrm{b} 3 \mathrm{c} 3}$ & $29.82 \pm 0.53^{\text {b3c3 }}$ \\
\hline \multirow{3}{*}{ NAL (cm) } & W 0 & $17.22 \pm 0.56$ & $17.81 \pm 0.52$ & $18.10 \pm 0.25$ & $17.88 \pm 0.22$ & $19.92 \pm 0.48$ & $17.74 \pm 0.30$ & $17.96 \pm 0.40$ \\
\hline & W13 & $20.40 \pm 0.24$ & $18.10 \pm 0.06^{\mathrm{a} 3}$ & $18.88 \pm 0.30^{\mathrm{a} 3}$ & $19.14 \pm 0.24^{\mathrm{a} 3}$ & $18.31 \pm 0.14^{\mathrm{a} 3}$ & $18.60 \pm 0.18^{\mathrm{a} 3}$ & $18.56 \pm 0.20^{\mathrm{a} 3}$ \\
\hline & W26 & $21.12 \pm 0.58$ & $17.98 \pm 0.28^{\mathrm{a} 3}$ & $18.12 \pm 0.07^{\mathrm{a} 3}$ & $20.12 \pm 0.37^{\mathrm{a} 2}$ & $19.32 \pm 0.32^{\mathrm{a} 2}$ & $19.55 \pm 0.18^{\mathrm{a} 2}$ & $19.22 \pm 0.77^{\mathrm{a} 2}$ \\
\hline \multirow{3}{*}{ AFM (g) } & W 0 & $1.25 \pm 0.56$ & $0.48 \pm 0.07$ & $1.42 \pm 0.24$ & $0.46 \pm 0.03$ & $1.51 \pm 0.16$ & $1.30 \pm 0.26$ & $1.32 \pm 0.08$ \\
\hline & W13 & $2.4 \pm 0.32$ & $9.87 \pm 0.42^{\mathrm{a} 3}$ & $10.65 \pm 0.79^{\mathrm{a} 3}$ & $8.81 \pm 0.28^{\mathrm{a} 3}$ & $10.78 \pm 0.74^{\mathrm{a} 3}$ & $8.87 \pm 0.48^{\mathrm{a} 3}$ & $8.88 \pm 0.79^{\mathrm{a} 3}$ \\
\hline & W26 & $2.32 \pm 0.32$ & $11.69 \pm 0.26^{\mathrm{a} 3}$ & $10.96 \pm 0.77^{\mathrm{a} 3}$ & $5.98 \pm 0.16^{\mathrm{a} 3 \mathrm{~b} 3 \mathrm{c} 3}$ & $6.65 \pm 6.65^{\mathrm{a} 3 \mathrm{~b} 3 \mathrm{c} 3}$ & $5.87 \pm 0.38^{\mathrm{a} 3 \mathrm{~b} 3 \mathrm{c} 3}$ & $6.47 \pm 0.41^{\mathrm{a} 3 \mathrm{~b} 3 \mathrm{c} 3}$ \\
\hline \multirow{3}{*}{$\operatorname{BMI}\left(\mathrm{g} / \mathrm{cm}^{2}\right)$} & W 0 & $0.44 \pm 0.01$ & $0.44 \pm 0.04$ & $0.40 \pm 0.02$ & $0.45 \pm 0.01$ & $0.35 \pm 0.013$ & $0.44 \pm 0.01$ & $0.43 \pm 0.02$ \\
\hline & W13 & $0.47 \pm 0.03$ & $0.64 \pm 0.02^{\mathrm{a} 3}$ & $0.60 \pm 0.02^{\mathrm{a} 3}$ & $0.57 \pm 0.01^{\mathrm{a} 3}$ & $0.55 \pm 0.01^{\mathrm{a} 3}$ & $0.61 \pm 0.01^{\mathrm{a} 3}$ & $0.56 \pm 0.02^{\mathrm{a} 3}$ \\
\hline & W26 & $0.46 \pm 0.01$ & $0.65 \pm 0.03^{\mathrm{a} 3}$ & $0.57 \pm 0.01^{\mathrm{a} 3}$ & $0.51 \pm 0.01^{\mathrm{b} 2 \mathrm{c} 2}$ & $0.48 \pm 0.01^{\mathrm{b} 2 \mathrm{c} 2}$ & $0.51 \pm 0.01^{\mathrm{b} 2 \mathrm{c} 2}$ & $0.51 \pm 0.02^{\mathrm{b} 2 \mathrm{c} 2}$ \\
\hline \multirow{3}{*}{$\begin{array}{l}\text { Lee Index } \\
\left(\mathrm{g} / \mathrm{cm}^{3}\right)\end{array}$} & W 0 & $0.29 \pm 0.00$ & $0.29 \pm 0.01$ & $0.28 \pm 0.01$ & $0.29 \pm 0.00$ & $0.26 \pm 0.01$ & $0.29 \pm 0.00$ & $0.29 \pm 0.01$ \\
\hline & W13 & $0.28 \pm 0.01$ & $0.33 \pm 0.00^{\mathrm{a} 3}$ & $0.32 \pm 0.00^{\mathrm{a} 3}$ & $0.31 \pm 0.00^{\mathrm{a} 3}$ & $0.31 \pm 0.00^{\mathrm{a} 3}$ & $0.32 \pm 0.00^{\mathrm{a} 3}$ & $0.31 \pm 0.00^{\mathrm{a} 3}$ \\
\hline & W26 & $0.27 \pm 0.01$ & $0.34 \pm 0.01^{\mathrm{a} 3}$ & $0.31 \pm 0.00^{\mathrm{a} 3}$ & $0.29 \pm 0.00^{\mathrm{a} 1 \mathrm{~b} 2 \mathrm{cl}}$ & $0.29 \pm 0.00^{\mathrm{a} 1 \mathrm{~b} 2 \mathrm{c} 1}$ & $0.30 \pm 0.00^{\mathrm{a} 1 \mathrm{~b} 2 \mathrm{c} 1}$ & $0.30 \pm 0.01^{\mathrm{alb} 2 \mathrm{cl}}$ \\
\hline \multirow{3}{*}{ FW (g/100g) } & W 0 & $1.30 \pm 0.10$ & $1.08 \pm 0.09$ & $1.43 \pm 0.09$ & $1.03 \pm 0.07$ & $1.21 \pm 0.07$ & $1.27 \pm 0.10$ & $1.14 \pm 0.13$ \\
\hline & W13 & $2.34 \pm 0.20$ & $1.00 \pm 0.04^{\mathrm{a} 3}$ & $0.99 \pm 0.06^{\mathrm{a} 3}$ & $1.25 \pm 0.04^{\mathrm{a} 3}$ & $1.42 \pm 0.18^{\mathrm{a} 3}$ & $1.10 \pm 0.09^{\mathrm{a} 3}$ & $1.31 \pm 0.13^{\mathrm{a} 3}$ \\
\hline & W26 & $2.60 \pm 0.07$ & $1.25 \pm 00.06^{\mathrm{a} 3}$ & $1.52 \pm 0.13^{\mathrm{a} 3}$ & $2.26 \pm 0.17^{\mathrm{b} 3 \mathrm{c} 3}$ & $2.97 \pm 0.10^{\mathrm{b} 3 \mathrm{c} 3}$ & $2.41 \pm 0.13^{\mathrm{b} 3 \mathrm{c} 3}$ & $2.74 \pm 0.06^{\mathrm{b} 3 \mathrm{c} 3}$ \\
\hline
\end{tabular}

G I: Normal control; G II: Satellite control; G III: oxidised palm oil and sucrose control; G IV and G VI: obese rats treated with pawpaw juice extract of Oblong form; G Vand VII: obese rats treated with pawpaw juice extract of Round form; AC: abdominal circumference, NAL: naso-anal length; AFM: abdominal fat mass; BMI: body mass index; Lee index; FW: faeces weight. Each value represent mean \pm Standard error, $\mathrm{n}=6$. $\mathrm{p}<0.05=$ significant difference; $\alpha, b$, c: significant difference when compared respectively with G I, G II and G III; a1/b1/c1 $=p<0.05, a 2 / b 2 / c 2=p<0.01, a 3 / b 3 / c 3=p<0.001$.

\subsection{Effects of C. Papaya on Oral Glucose Tolerance Test, Insulin Sensitivity and Insulin Sensitivity Index}

It was observed that, concomitant oral administration of the juice fruit (all varieties of $C$. papaya) with OPOS diet inhibited significantly $(\mathrm{P}<0.001)$ the increase of area under curve of oral glucose tolerance test with a minimal on G IV group (243.13 versus 459.3; 201.05 versus 474.15 respectively for males and females) compared to G II groups. However, a significant difference $(\mathrm{P}<0.001)$ was observed between G I group and groups receiving C. papaya fruit extract. The administration of juice fruit of all varieties with OPOS diet provoked a significant $(\mathrm{P}<0.001)$ decrease of area under curve of insulin sensitivity with a minimal on $G$ IV
(316.05 versus 459.05 ; 308.1 versus 474.15 respectively for males and females) compared to OPOSC groups. On the other hand, a supplement of oxidised palm oil and sucrose induced a significant decrease $(\mathrm{p}<0.001)$ of insulin sensitivity index of $63.64 \%$ and $85.32 \%$ respectively for males and females compared to G I group (Figure 2). Moreover, all varieties of $\mathrm{C}$. papaya juice extract administered in association with OPOS $+\mathrm{S}$ diet induced a significant increase $(p<0.001)$ in the insulin sensitivity index with a maximal on G IV group in males ( 0.51 versus 0.16$)$, on $\mathrm{G} \mathrm{V}$ in females $(0.39$ versus 0.09$)$ compared to G II groups. The inhibition was $218.75 \%$ and by $333.33 \%$ respectively for males and females. No significant difference of pawpaw juice effect $(\mathrm{P}>0.05)$ was observed neither between oblong and round form groups, neither between animals fed with 
normal diet and those fed with OPOS, nor between males and females (Figures 1, 2, 3).

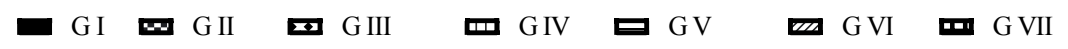

A

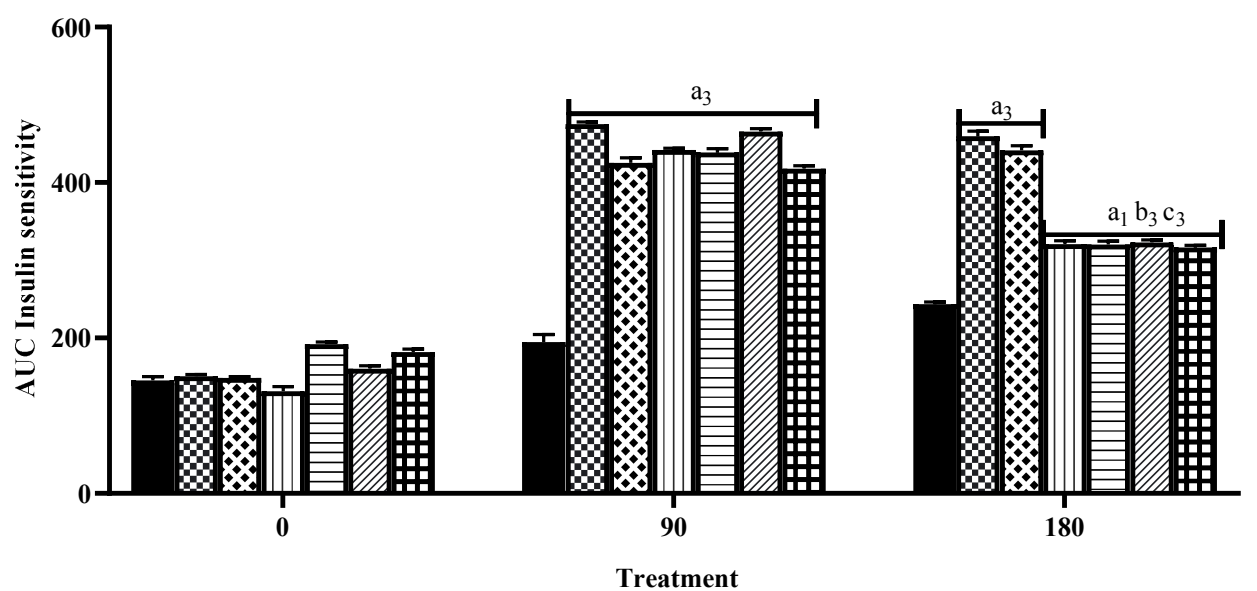

B

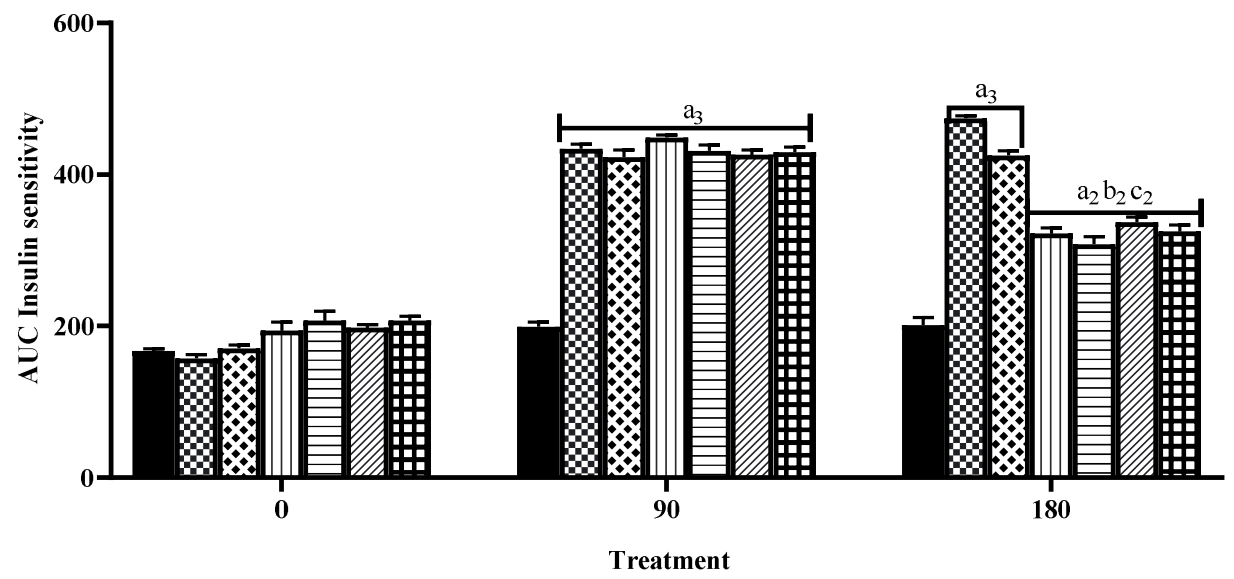

Figure 1. Aera under curve of insilin sensitivity in animals during period.

A: Males; B: Females; G I: Normal control; G II: Satellite control; G III: oxidised palm oil and sucrose control; G IV and G VI: obese rats treated with pawpaw juice extract of Oblong form; G Vand VII: obese rats treated with pawpaw juice extract of Round form. Each value represent mean \pm Standard error, $\mathrm{n}=6$. $\mathrm{p}<0.05=$ significant difference; $\alpha, \mathrm{b}$, c: significant difference when compared respectively with G I, G II and G III; $\mathrm{a} 1 / \mathrm{b} 1 / \mathrm{c} 1=\mathrm{p}<0.05, \mathrm{a} 2 / \mathrm{b} 2 / \mathrm{c} 2=\mathrm{p}<0.01$, $\mathrm{a} 3 / \mathrm{b} 3 / \mathrm{c} 3=\mathrm{p}<0.001$.

A

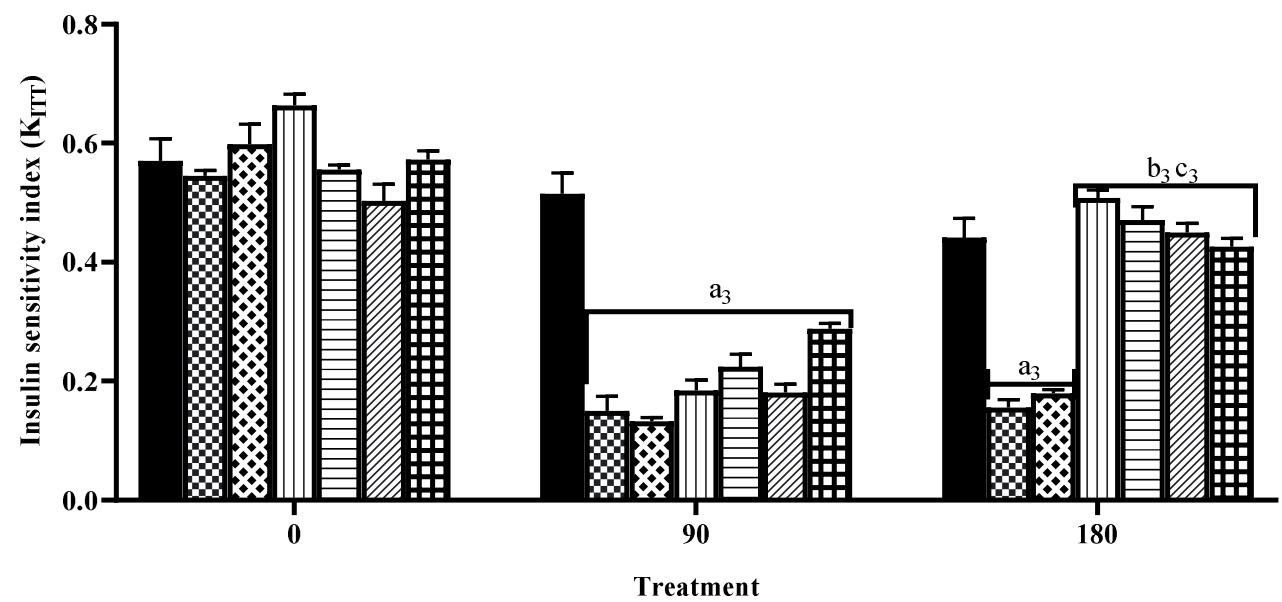




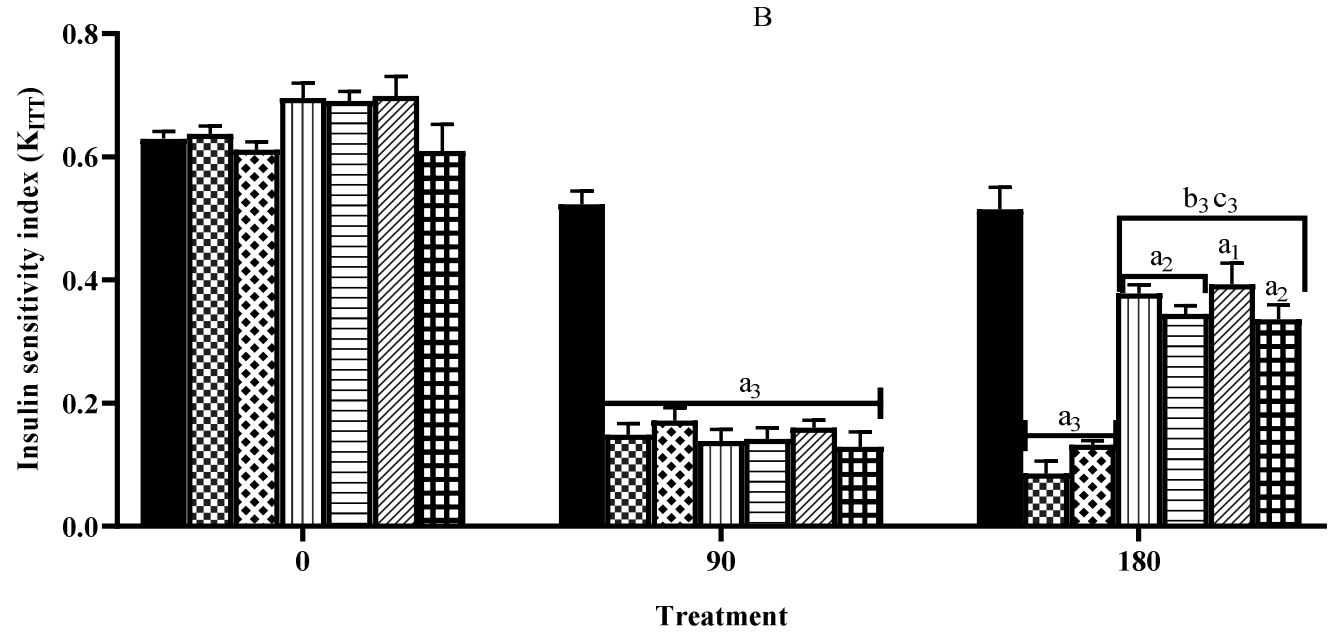

Figure 2. Insulin sensitivity index in animals during experiment period.

A: Males; B: Females; G I: Normal control; G II: Satellite control; G III: oxidised palm oil and sucrose control; G IV and G VI: obese rats treated with pawpaw juice extract of Oblong form; G Vand VII: obese rats treated with pawpaw juice extract of Round form. Each value represent mean \pm Standard error, $\mathrm{n}=6$. $\mathrm{p}<0.05=$ significant difference; $\alpha, \mathrm{b}$, c: significant difference when compared respectively with G I, G II and G III; $\mathrm{a} 1 / \mathrm{b} 1 / \mathrm{c} 1=\mathrm{p}<0.05, \mathrm{a} 2 / \mathrm{b} 2 / \mathrm{c} 2=\mathrm{p}<0.01$, $\mathrm{a} 3 / \mathrm{b} 3 / \mathrm{c} 3=\mathrm{p}<0.001$.

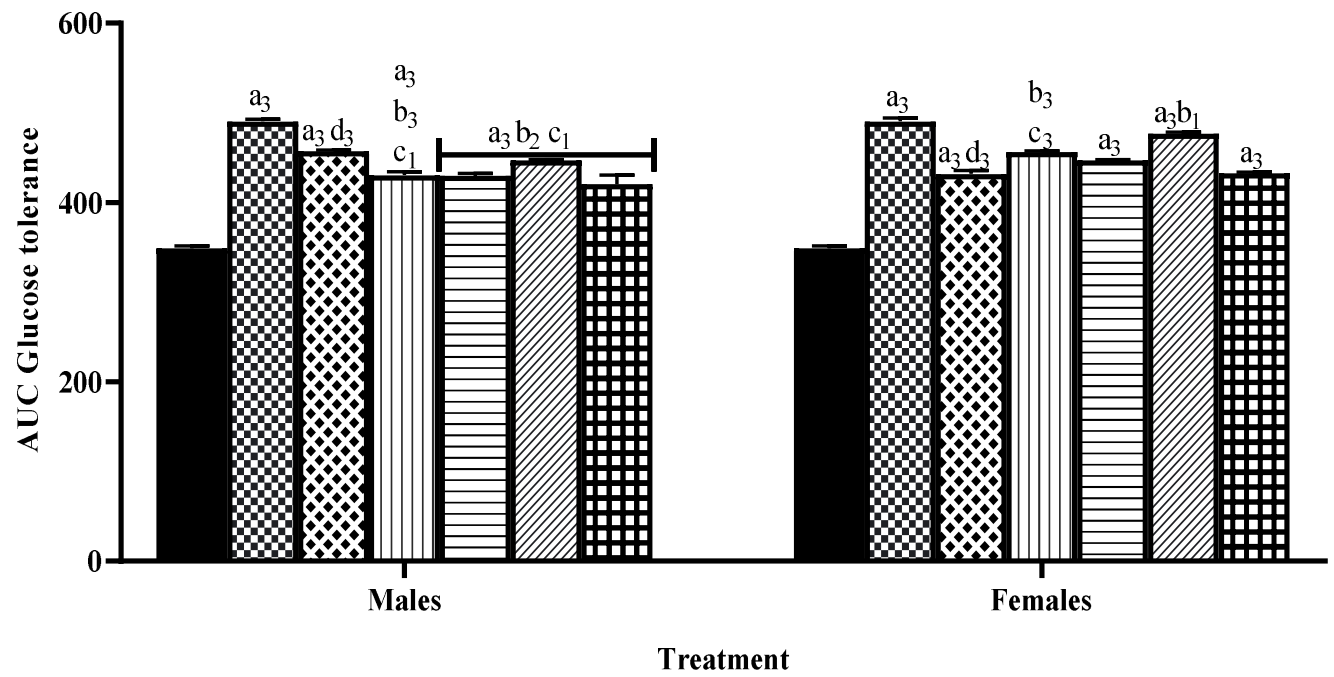

Figure 3. Aera under curve of glucose tolerance in animals after treatment.

G I: Normal control; G II: Satellite control; G III: oxidised palm oil and sucrose control; G IV and G VI: obese rats treated with pawpaw juice extract of Oblong form; G Vand VII: obese rats treated with pawpaw juice extract of Round form. Each value represent mean \pm Standard error, $n=6$. $p<0.05=$ significant difference; $\alpha$, b, c: significant difference when compared respectively with G I, G II and G III; a1/b1/c1=p<0.05, a2/b2/c2=p<0.01, $\mathrm{a} 3 / \mathrm{b} 3 / \mathrm{c} 3=\mathrm{p}<0.001$.

\subsection{Effects of Some Varieties of A. Comosus on Serum Lipid Profile}

All varieties of $C$. papaya juice fruit extract administered during the last 13 weeks of experimental period induced a decrease of triglycerides, total cholesterol, LDL-c and atherogenic index as compared to the OPOS control. The decrease in triglycerides maximal was 54.75 and $53.32 \%$ in G V group (173.3 versus 394.9 and 173 versus 347.5 respectively for males and females). On the other hand, serum total cholesterol decreased with a maximal of $51.08 \%$ and $40.28 \%$ in $\mathrm{G} \mathrm{V}$ respectively for males and females, LDL- c by $64.4 \%$ and $54.83 \%$ in the same groups respectively for males and females. HDL-c which was significantly high of $61.47 \%$ and $60.41 \%$ (respectively for males and females) compared to animals receiving OPOS diet. A significant increase in atherogenic index was observed when compared to normal control. The treatment with all varieties juice fruit receiving OPOS diet significantly reduced these parameters near normal values range. No significant difference of pawpaw juice effect $(\mathrm{P}>0.05)$ was observed neither between oblong and round form groups, neither between animals fed with normal diet and those fed with OPOS, nor between males and females (Table 2). 
Table 2. Effects of concomitant administration of Carica papaya (Oblong and Round form) juice extract and oxidised palm oil +sucrose on lipid profile, atherogenic index and insulin resistance in rats.

\begin{tabular}{|c|c|c|c|c|c|c|c|}
\hline Groups & G I & G II & G III & G IV & G V & G VI & G VII \\
\hline & MALES & & & & & & \\
\hline TG & $150.8 \pm 10.98$ & $394.9 \pm 14.64^{\mathrm{a} 3}$ & $287 \pm 16.75^{\mathrm{a} 3}$ & $178,7 \pm 8,25^{\text {b3c3 }}$ & $194,4 \pm 10,15^{\text {b3c3 }}$ & $195,1 \pm 5,96^{\mathrm{b3c3}}$ & $178,3 \pm 3,19^{\mathrm{b} 3 \mathrm{c} 3}$ \\
\hline HDL-c & $92.7 \pm 1.81$ & $35.35 \pm 1.42^{\mathrm{a} 3}$ & $41.6 \pm 1.08^{\mathrm{a} 3}$ & $91,75 \pm 1,6^{\mathrm{b} 3 \mathrm{c} 3}$ & $91,15 \pm 1,71^{\text {b3c3 }}$ & $75,6 \pm 4,31^{\text {alb3c3 }}$ & $83,35 \pm 3,46^{\mathrm{b3c} 3}$ \\
\hline $\mathrm{TC}$ & $393 \pm 2.29$ & $865.9 \pm 73.61^{\mathrm{a} 3}$ & $810.3 \pm 83.29^{\text {a3 }}$ & $423,6 \pm 7,367^{\mathrm{b} 3 \mathrm{c} 3}$ & $423 \pm 23,32^{\mathrm{b} 3 \mathrm{c} 3}$ & $491,6 \pm 19,37^{\mathrm{b} 3 \mathrm{c} 3}$ & $470,2 \pm 23,24^{\mathrm{b} 3 \mathrm{c} 3}$ \\
\hline LDL & $270.9 \pm 2.7$ & $768.9 \pm 99.54^{\mathrm{a} 3}$ & $704.5 \pm 101.5^{\text {a3 }}$ & $296,6 \pm 12,06^{\mathrm{b} 3 \mathrm{c} 3}$ & $273,7 \pm 27,63^{\mathrm{b} 3 \mathrm{c} 3}$ & $378,2 \pm 24,37^{\mathrm{b} 3 \mathrm{c} 3}$ & $363,3 \pm 18,43^{\mathrm{b} 3 \mathrm{c} 3}$ \\
\hline AI & $4.25 \pm 0.06$ & $25.02 \pm 2.81^{\mathrm{a} 3}$ & $19.47 \pm 2.74^{\mathrm{a} 3}$ & $4,63 \pm 0,18^{\mathrm{b} 3 \mathrm{c} 3}$ & $4,43 \pm 0,32^{\mathrm{b} 3 \mathrm{c} 3}$ & $6,55 \pm^{\text {b3c3 }}$ & $5,79 \pm 0,07^{\mathrm{b} 3 \mathrm{c} 3}$ \\
\hline \multirow[t]{2}{*}{ IR } & $1.63 \pm 0.12$ & $11.28 \pm 0.79^{\mathrm{a} 3}$ & $6.9 \pm 0.35^{\mathrm{a} 3}$ & $1,95 \pm 0,07^{\text {b3с3 }}$ & $2,13 \pm 0,08^{\mathrm{b} 3 \mathrm{c} 3}$ & $2,62 \pm 0,18^{\mathrm{b3c3}}$ & $2,15 \pm 0,06^{\mathrm{b3c3}}$ \\
\hline & FEMALES & & & & & & \\
\hline TG & $151.1 \pm 9.40$ & $347.5 \pm 35.12^{a 3}$ & $277.1 \pm 12.06^{\mathrm{a} 3}$ & $177,7 \pm 6,23^{\mathrm{b3c3}}$ & $173 \pm 10,42^{\mathrm{b3c3}}$ & $181,6 \pm 1,68^{\mathrm{b3c} 3}$ & $182,6 \pm 5,71^{\text {b3c3 }}$ \\
\hline HDL-c & $85.65 \pm 3.53$ & $37.65 \pm 0.89^{\mathrm{a} 3}$ & $43.55 \pm 1.85^{\text {а3 }}$ & $97,75 \pm 1,61^{\text {b3c3 }}$ & $121,9 \pm 1,26^{\text {азьb3c3 }}$ & $95,10 \pm 4,38^{\mathrm{b} 3 \mathrm{c} 3}$ & $101,9 \pm 1,82^{\mathrm{a} 2 \mathrm{~b} 3 \mathrm{c} 3}$ \\
\hline $\mathrm{TC}$ & $387.6 \pm 5.31$ & $707.4 \pm 47.63^{\text {a3 }}$ & $577.6 \pm 26.03^{\text {a } 3}$ & $422,9 \pm 10,36^{\mathrm{b3c3}}$ & $431,8 \pm 9,08^{\mathrm{b} 3 \mathrm{c} 3}$ & $470,1 \pm 25,72^{\mathrm{b3cl}}$ & $423,8 \pm 23,89^{\mathrm{b} 3 \mathrm{c} 3}$ \\
\hline LDL & $269.8 \pm 8.38$ & $598.2 \pm 68.37^{\text {a3 }}$ & $457 \pm 15.9^{\mathrm{a3}}$ & $295,2 \pm 11,88^{\mathrm{b} 3 \mathrm{c} 2}$ & $270,2 \pm 12,24^{\mathrm{b} 3 \mathrm{c} 3}$ & $308,7 \pm 25,57^{\mathrm{b3c} 2}$ & $265 \pm 28,12^{\text {b3c3 }}$ \\
\hline AI & $4.54 \pm 0.22$ & $18.76 \pm 1.84^{\mathrm{a} 3}$ & $12.79 \pm 0.21^{\mathrm{a} 3}$ & $4,39 \pm 0,12^{\mathrm{b} 3 \mathrm{c} 3}$ & $3,5 \pm 0,1^{\text {b3c3 }}$ & $4,68 \pm 0,36^{\mathrm{b} 3 \mathrm{c} 3}$ & $3,93 \pm 0,28^{\mathrm{bcc} 3}$ \\
\hline IR & $1.77 \pm 0.11$ & $9.34 \pm 1.15^{\mathrm{a} 3}$ & $6.42 \pm 0.41^{\mathrm{a} 3}$ & $1,82 \pm 0,09^{\text {b3c } 3}$ & $1,42 \pm 0,08^{\mathrm{b} 3 \mathrm{c} 3}$ & $1,92 \pm 0,09^{\mathrm{b} 3 \mathrm{c} 3}$ & $1,79 \pm 0,05^{\mathrm{b3c3}}$ \\
\hline
\end{tabular}

G I: Normal control; G II: Satellite control; G III: oxidised palm oil and sucrose control; G IV and G VI: obese rats treated with pawpaw juice extract of Oblong form; G Vand VII: obese rats treated with pawpaw juice extract of Round form; TC: total cholesterol, TG: triglyceride; HDL-C: high-density lipoprotein cholesterol; LDL-C: low-density lipoprotein cholesterol; AI: atherogenic index; IR: insulin resistance. Each value represent mean \pm Standard error, Unit is $\mathrm{mg} / \mathrm{dL} . \mathrm{n}=6 . \mathrm{p}<0.05=$ significant difference; $\alpha, \mathrm{b}$, c: significant difference when compared respectively with G I, G II and G II; a1/b1/c1=p<0.05, $\mathrm{a} 2 / \mathrm{b} 2 / \mathrm{c} 2=\mathrm{p}<0.01, \mathrm{a} 3 / \mathrm{b} 3 / \mathrm{c} 3=\mathrm{p}<0.001$.

\section{Discussion}

Our study shows that the administration of OPOS diet induced metabolic disorders including obesity, dyslipidemia, and consequently insulin resistance [14, 15]. Continual heating of palm oil became harmful to organism. Actually, palm oil used repetitively induces fatty acids oxidation, and increases the rate of cytotoxic fatty acid accumulation, resulting in the alteration of insulin response and hypertension [16]. Moreover, a diet rich in sucrose results in insulin resistance [17]. Oxidised palm oils combinated to sucrose could react synergically to cause abnormalities including glucose tolerance, insulin resistance and hyperlipidemia. This study investigation showed that, standard diet associated with oxidised palm oil and sucrose during 13 weeks induced an increase of abdominal circumference, BMI and Lee index. This would be due to the presence of fructose, oleic and palmitic acid within the diet. It was also observed an increase of abdominal fat strengthened by the lipogenic character of the diet that explain the hypertrophy and herplasia of adipocytes. Animals receiving OPOS diet presented hyperglycemia, glucose intolerance and insulin insensibility. Sucrose can be metabolized into glucose and fructose which may be transformed into lipid through lipogenesisde novo pathway and in turn induce lipotoxixityandglucotoxicity; preventing insulin action or secretion at cellular levels resulting in a hyperglycemia [18, 19]. In addition, oxidised palm oil increase the level of mono and polyunsaturated fatty acids and thus increase the rate of fats. Thus oxidised palm oil and sucrose contribute to cytotoxic fatty acid accumulation and the alteration of insulin response [16]. The insulin resistance observed in this study is related to the increase of abdominal fats $[18,20]$ and corroborate the insulin sensitivity index $\left(\mathrm{K}_{\mathrm{itt}}\right)$ which was dramatically dropped in animals receiving OPOS diet. Administration of juice fruits extracts of all varieties of $C$. papaya with OPOS diet inhibited an increase of blood glucose levels in OGTT (oral glucose tolerance test) and IST (insulin sensitivity test). The benefits of $C$. papaya on glycemia are reported by JuárezRojop et al. [21]. This could be due to the actions of our fruits extracts at peripheral levels by reducing glucose absorption from the gastrointestinal tract and/or stimulating peripheral glucose utilization [22, 23]. Moreover, during thirteen weeks of treatment with oblong and round form of pawpaw fruits, we also observed hypoglycemic effects that were similar to those obtain by Juárez-Rojop et al. [21]. It should be due to probable stimulatory mechanism on the few surviving $\beta$-cells, which could allow the release of more insulin [21-24]. Our study suggests that C. papaya juice fruit may act by stimulating the few remaining $\beta$-cells with release of insulin. Pawpaw fruits extracts (oblong and round form) after thirteen weeks of treatment involved a decrease of serumlevel of triglycerides, total cholesterol, LDLc and an increase of serum level of HDLc. These results are involved in prevention of development atherosclerosis and coronary heart disease and similar to those of Zetina-Esquivel et al. (2015) [25], and similar results have already been reported [26, 27]. These fruits could modulate blood lipid abnormalities, suggesting that, fruit would be helpful to prevention of obesity and comorbidities by reduction of dyslipidemia. Juice fruit extract could modulate blood lipid abnormalities, suggesting that, all varieties of $C$. papaya in this study would be helpful to the prevention of obesity and complications through reduction of dyslipidemia, amelioration of insulin sensitivity. Moreover, previous studies have reported that some phytocomponents as saponins, steroids contents in Carica papaya endowed antihyperlipidemic and antiatherogenic action by inhibiting intestinal lipid absorption via resin-like action and 
inhibition of lipase activity [21]. These effects could be due to the decreases of intestinal absorption of cholesterol beside the reabsorption of bile acids and neutral sterol [26]. The effects of these varieties of Carica papaya could be due to the existence of papain that having lipolytic and proteolytic activity and to the contents of high amount of fiber and polyphenol compound in raw juice which induced hypocholesterolemic effects [10].

\section{Conclusion}

A supplement of oxidised palm oil (25\%) and sucrose $(25 \%)$ during thirteen weeks induced metabolic alteration. Treatment with the two varieties - oblong and round forms of pawpaw considerably improved glucose tolerance, insulin sensitivity, and lipidaemia, and ameliorated anthropometrical parameters. Pawpaw should then be recommended by nutritionist against metabolic diseases.

\section{Recommendations}

Oxidized oils and sugar consumption drastically negatively affect cardiometabolic health. As a functional food, Pawpaw juice regular consumption can provide major benefits on many of these cardiometabolic abnormalities, and then either treat, prevent or improve cardiometabolic risk, no matter if the diet is safe or not. Nevertheless, adopting a safe diet gives better results than unsafe one. Generally, the use of foods rich in micronutrient regulates metabolic disorders and help to improve non-communicable diseases.

\section{Conflicts of Interest}

The authors declare that they have no competing interests.

\section{Acknowledgements}

The authors are thankful to the Research Unit of Animal Phytopharmacology and Biochemistry of the University of Douala for material support.

\section{References}

[1] Alberti, K., Eckel, R. H., Grundy, S. m., Zimmet, P. Z., Cleeman, J. L., Donato, K. A., Fruchart J. C., James, W. P. T., Loria, C. M., Smith, S. C. Harmonizing the metabolic syndrome a joint interim statement of the international diabetes federation task force on epidemiology and prevention; national heart, lung and blood institute; American heart association; world heart federation; international atherosclerosis society; and international association for the study of obesity. 2009. Circulation. 120, 1640-1645.

[2] Lyons, C., Kennedy, E. Roche, H. Metabolic inflammationdifferential modulation by dietary constituents. 2016 . Nutrients. 8, 247.

[3] Freedman, D. Obesity-United States, 1988-2008. MMWR Surveillance Summaries 60. Suppl 2011. 73-77.
[4] Peters, A. J., Barendregt, F., Willekens, et al. Obesity in adulthood and its consequences for life expectancy: A lifetable analysis. Annals of Internal Medicine. 2003. 138: 11381145 .

[5] Grundy, S. M. Obesity, metabolic syndrome and cardiovascular disease. J. Clin. Endocrinol. Metab. 2004. 89, 2595-2600.

[6] Clarke, S. D. Polyunsaturated fatty acid regulation of gene transcription: A mechanism to improve energy balance and insulin resistance. Br. J. Nutr. 2000. 83, S59-S66.

[7] Simopoulos, A. P. An increase in the $\omega-6 / \omega-3$ fatty acid ratio increases the risk for obesity. Nutrients. 2016. 8, 128.

[8] Fontana, L. Modulating human aging and ageassociated diseases. Biochimicaet Biophysica Acta 1790: 1133-1138. doi: 10. 1016/j. bbagen. 2009. 02. 002.

[9] Colquitt, J. L., Pickett, K., Loveman, E., and Frampton G. K. Surgery for weight loss in adults. The Cochrane Database of Systematic ReviewsCD003641. 2014. doi: 10. 1002/14651858. CD003641. pub4.

[10] Santana L. F., Inada A. C., Spontoni do Espirito Santo B. L., Filiú, Arnildo Pott W. F. O., Alves F. M., Guimarães R. C. A., Freitas K. C., and Hiane P. A. Nutraceutical Potential of Carica papayain Metabolic Syndrome. Nutrients. 2019. 11, 1608; doi: 10. 3390/nu1107160.

[11] Ngueguim F. T., Esse E. C., DzeufietD. P. D., GounoueK. R., Bilanda D. C., Kamtchouing P. and DimoT. Oxidised palm oil and sucrose induced hyperglycemia in normal rats: effects of Sclerocaryabirreastem barks aqueous extract BMC Complement and Altern Med. 2016. 16: 47 DOI 10. 1186/s12906-016-1009-0.

[12] Lindsey G., Fida B., SoJung L., Hala T., Elisa A., Silva A. Surrogate Estimates of Insulin Sensitivity in Obese Youth along the Spectrum of Glucose Tolerance from Normal to Prediabetes to Diabetes. J Clin Endocrinol Metab. 2011. 96 (7): 2136-45.

[13] Wu T. T., Gao Y., Zheng Y. Y., Yi-Tong Ma., and Xie X. atherogenic index of plasma (AIP): a novel predictive indicator for the coronary artery disease in postmenopausal women. Lipids in Health and Disease. 2018. 17: 197.

[14] Bilanda D. C., Dimo T., Djomeni D. D. P., Bella T. M. N., Aboubakar F. B. O., Nguelefack B. T., et al. Antihypertensive and antioxidant effects ofAllanblackia floribunda Oliv. (Clusiaceae) aqueous extract in alcohol- and sucrose-induced hypertensive rats. J Ethnopharmacol. 2010. 128: 634-40.

[15] Hermier D. Impact métabolique des acides gras saturés/insaturés. Innovations Agronomiques. 2010; 10: 11-23. In French.

[16] Roberts C. K., Barnard R. J., Sinhu R. K., Ehdai A., Vaziri N. D. Oxidative stress and dysregulation of NAD (P)H oxidase and antioxidant enzymes in diet-induced metabolic syndrome. Metabolism. 2006. 55: 928-34.

[17] Mahsa M., Yong M. G., Mahdi E. Metabolic effects of high sucrose and saturated oil feeding on insulin resistance in Sprague-Dawley rats. Indian j exp Biol. 2015. 53: 264-72.

[18] Scheen A. J., Lefèbvre P. J. Pathophysiology of Type 2 diabetes. In: Springer Verlag Berlin, editor. Oral Antidiabetics. 1996. p. 7-42. 
[19] Unger R. H. Lipotoxicity in the pathogenesis of obesitydependent NIDDM: Genetic and clinical implications. Diabetes. 1995. 44 (8): 863-70.

[20] Nawel H., Bénédict B., Catherine C., Abdel N. A., Philip R., Henri G., et al. Prevention of type 2 diabetes induced by high fat diet in the C57BL/6J mouse by two medicinal plants used in traditional treatment of diabetes in the east of Algeria. $\mathrm{J}$ Ethnopharmacol. 2010. 128: 513-8.

[21] Juárez-Rojop I. E., Díaz-Zagoya J. C., Ble-Castillo J. L., Miranda-Osorio P. H., Castell-Rodríguez A. E., Tovilla-Zárate C. A., Rodríguez-Hernández A., Aguilar-Mariscal H., RamónFrías T., and Bermúdez-Ocaña D. Y. Hypoglycemic effect of Caricapapaya leaves in streptozotocin-induced diabetic rats. BMC Complement and Altern Med. 2012. 12: 236 http://www.biomedcentral.com/1472-6882/12/236

[22] Abeywickrama K. R., Ratnasooriya W. D., Amarakoon, A. M. Oralhypoglycaemic, antihyperglycaemic and antidiabetic activities of Sri Lankan broken orange pekoe fannings (BOPF) grade black tea (Camellia sinensisL.) in rats. J Ethnopharmacol. 2011. 135: 278-286.

[23] Baquer N. Z., Kumar P., Taha A., Kale R. K., Cowsik S. M., McLean P. Metabolic and molecular action of Trigonellafoenum-graecum (fenugreek) and trace metals in experimental diabetic tissues. J Biosci. 2011. 36: 383-396.
[24] Cumaoğlu A., Ari N., Kartal M., Karasu Ç. Polyphenolic extracts from Oleaeuropea L. protect against cytokineinduced $\beta$-cell damage through maintenance of redox homeostasis. Rejuvenation Res. 2011. 14: 325-334.

[25] Alma M., Zetina-Esquivel., Carlos A., Tovilla-Zárate, Crystell Guzmán-Garcia, Rodríguez-Hernández, A., Andrés E., Castell-Rodríguez, Jorge L., Ble-Castillo, Avila-Fernandez, A., Isela E., Juárez-Rojop, Juan C., Díaz-Zagoya. Effect of Carica papaya Leaf Extract on Serum Lipids and Liver Metabolic Parameters of Rats Fed a High Cholesterol Diet Health. 2015. 7, 1196-1205.

[26] Pronin, A. V., Danilov, L. L., Narovlyansky, A. N. and Sanin, A. V. Plant Polyisoprenoids and Control of Cholesterol Level. ArchivumImmunologiaeetTherapiaeExperimentalis. 2014. 62, 31-39. http://dx. doi. org/10. 1007/s00005-013-0253-y

[27] Anandhi, R., Annadurai, T., Anitha, T., Muralidharan, A. and Najmunnisha, K. Antihypercholesterolemic and Antioxidative Effects of an Extract of the Oyster Mushroom, Pleurotusostreatus, and Its Major Constituent, Chrysin, in Triton WR-1339-Induced Hypercholesterolemic Rats. J $\begin{array}{llll}\text { PhysiolBiochem. } & 2013 . & 69, & 313-323 .\end{array}$ http://dx.doi.org/10.1007/s13105-012-0215-6. 